OPEN ACCESS

Edited by: Fumito Maruyama,

Kyoto University, Japan

Reviewed by:

María de Toro,

Centro de Investigación Biomédica de La Rioja (CIBIR), Spain

Miklos Fuzi,

Semmelweis University, Hungary

*Correspondence:

Marie A. Chattaway

marie.chattaway@phe.gov.uk

Specialty section:

This article was submitted to Infectious Diseases,

a section of the journal

Frontiers in Microbiology

Received: 21 June 2017

Accepted: 23 August 2017

Published: 19 September 2017

Citation:

Chattaway MA, Greig DR, Gentle A,

Hartman HB, Dallman TJ and

Jenkins C (2017) Whole-Genome

Sequencing for National Surveillance

of Shigella flexneri.

Front. Microbiol. 8:1700.

doi: 10.3389/fmicb.2017.01700

\section{Whole-Genome Sequencing for National Surveillance of Shigella flexneri}

\author{
Marie A. Chattaway*, David R. Greig, Amy Gentle, Hassan B. Hartman, \\ Timothy J. Dallman and Claire Jenkins
}

Gastrointestinal Bacteria Reference Unit, Public Health England, London, United Kingdom

National surveillance of Shigella flexneri ensures the rapid detection of outbreaks to facilitate public health investigation and intervention strategies. In this study, we used whole-genome sequencing (WGS) to type S. flexneri in order to detect linked cases and support epidemiological investigations. We prospectively analyzed 330 isolates of $S$. flexneri received at the Gastrointestinal Bacteria Reference Unit at Public Health England between August 2015 and January 2016. Traditional phenotypic and WGS sub-typing methods were compared. PCR was carried out on isolates exhibiting phenotypic/genotypic discrepancies with respect to serotype. Phylogenetic relationships between isolates were analyzed by WGS using single nucleotide polymorphism (SNP) typing to facilitate cluster detection. For 306/330 (93\%) isolates there was concordance between serotype derived from the genome and phenotypic serology. Discrepant results between the phenotypic and genotypic tests were attributed to novel O-antigen synthesis/modification gene combinations or indels identified in O-antigen synthesis/modification genes rendering them dysfunctional. SNP typing identified 36 clusters of two isolates or more. WGS provided microbiological evidence of epidemiologically linked clusters and detected novel O-antigen synthesis/modification gene combinations associated with two outbreaks. WGS provided reliable and robust data for monitoring trends in the incidence of different serotypes over time. SNP typing can be used to facilitate outbreak investigations in real-time thereby informing surveillance strategies and providing the opportunities for implementing timely public health interventions.

Keywords: Shigella flexneri, whole-genome sequencing, surveillance, outbreaks, phylogeny

\section{INTRODUCTION}

Shigellosis is caused by four species of Shigella, including S. boydii, S. dysenteriae, S. flexneri and S. sonnei, transmitted via the fecal oral route. Symptoms typically start 1-2 days after exposure and include diarrhea, bloody diarrhea abdominal pain, fever, and tenesmus. The burden of shigellosis is highest in developing countries with up to 167 million episodes of diarrhea annually, leading to over a million deaths (Kotloff et al., 1999). A multicenter study of shigellosis in six Asian countries indicated the incidence rate to be highest in children under the age of 4 years old and in adults over 70 years old (von Seidlein et al., 2006). In the United Kingdom, S. flexneri is most commonly associated with causing travelers' diarrhea and outbreaks of gastrointestinal symptoms in men 
who have sex with men (MSM) (Simms et al., 2015). Furthermore, there are reports of increased intercontinental dissemination of multidrug resistant S. flexneri (Baker et al., 2015).

Between 2004 and 2015, 18,266 Shigella cases were reported by GBRU for England and Wales with S. flexneri accounting for 7075 (39\%) of these infections ( $S$. sonnei $n=8897,49 \%$; S. boydii $n=1364,7 \%$; S. dysenteriae $n=808,4 \%$; Shigella species unknown $n=122,1 \%$ ).

Shigella flexneri are traditionally serotyped phenotypically using antisera raised in rabbits, although molecular PCR methods have been implemented in a number of reference laboratories (Zhang et al., 2012; Gentle et al., 2016). Serotyping provides limited resolution with serotypes $2 \mathrm{a}(n=2448,35 \%)$, 3a $(n=1476,21 \%), 6(n=1047,15 \%)$, and $1 \mathrm{~b}(n=711$, $10 \%$ ) accounting for $81 \%$ of $S$. flexneri cases. Without a higher level of discrimination, outbreak detection is dependent on the identification of epidemiological links between cases belonging to the same serotype.

Whole-genome sequencing (WGS) has been shown to have potential in replacing traditional phenotypic and PCR methods (Gentle et al., 2016) for routine surveillance. This approach has the added value of further discriminating strains by their genetic relatedness to a single nucleotide polymorphism (SNP) level and has been used to investigate multiple gastrointestinal outbreaks at Public Health England (PHE) (McDonnell et al., 2013; Dallman et al., 2015, 2016).

The aim of this study was to compare traditional serotyping with WGS for serotyping S. flexneri for routine public health surveillance and to evaluate utility of WGS data to support epidemiologically linked clusters and outbreak investigations.

\section{MATERIALS AND METHODS}

\section{Bacterial Strains}

Bacterial isolates of S. flexneri from 330 cases were submitted to the Gastrointestinal Bacterial Reference Unit between August 2015 and January 2016, from local and regional hospital laboratories in England and Wales. This strain set comprised the following phenotypic serotypes (numbers of isolates belonging to each serotype in parenthesis): 1a (3), 1b (16), 1c (25), 2a (185), 2b (16), 3a (41), 3b (7), 4av/E1037 (9), 6 (19) X (4), Y (4), X and Y (1). Epidemiological data on age, sex, and region of residence were available from laboratory report forms. Travel history was available for 176 of cases. All isolates were serotyped and WGS.

\section{Serotyping and PCR}

Phenotypic identification of $S$. flexneri isolates were confirmed using the Omnilog GenIII MicroPlate (Biolog, Hayward, CA, United States). Serotyping was carried out using standard methods by slide agglutination using both commercially available monovalent antisera (Denka Seiken, Japan) and monoclonal antibody reagents (Reagensia AB, Sweden) and in-house antisera raised in rabbits (Gross and Rowe, 1985). Molecular PCR was carried out on the discrepancies between phenotypic and WGS typing results as previously described (Gentle et al., 2016) and a serotype assigned according to the gene combination detected (Supplementary Table S1).

\section{Whole-Genome Sequencing}

Genome sequencing and sequencing analysis were carried out as previously described (Dallman et al., 2015). Genomic DNA extracted using the QiaSymphony DNA extraction platform (Qiagen) from 330 S. flexneri was fragmented and tagged for multiplexing with Nextera XT DNA Sample Preparation Kits (Illumina) and sequenced using the Illumina HiSeq 2500 at PHE. A reference database containing the gene sequences encoding the $12 \mathrm{O}$-antigen synthesis or modification genes described by Sun et al. (2011, 2012a,b), including wzxc1-5, wzxe1-5, wzx6, gtrI, gtrII, gtrIV, gtrV, gtrX, gtrlc, oac oac1b and opt, was constructed. Using the GeneFinder tool (Doumith, unpublished), FASTQ reads were mapped to the $S$. flexneri $\mathrm{O}$-antigen synthesis or modification genes using Bowtie 2 (Langmead and Salzberg, 2012) and the best match to each target was reported with metrics including coverage, depth, mixture and nucleotide similarity in XML format for quality assessment. Only in silico predictions of serotype that matched to a gene determinant at $>80 \%$ nucleotide identity over $>80 \%$ target gene length were accepted. FASTQ sequences were deposited in the National Center for Biotechnology Information Short Read Archive under the bioproject PRJNA315192 (see Supplementary Table S2 for SRA identifiers).

\section{Cluster Detection}

Short reads were quality trimmed and mapped to the reference S. flexneri serotype 2a strain 2457T (AE014073.1) (Wei et al., 2003) or the reference strain NC_007613 if S. flexneri serotype 6, using BWA v0.75 (Li et al., 2009; Bolger et al., 2014). The Sequence Alignment Map output from BWA was sorted and indexed to produce a Binary Alignment Map (BAM) using Samtools (Li and Durbin, 2010). GATK v2.6.5 was used to create a Variant Call Format (VCF) file from each of the BAMs, which were further parsed to extract only SNP positions which were of high quality $(\mathrm{MQ}>30$, DP > 10, GQ > 30, Variant Ratio > 0.9) (McKenna et al., 2010). Gubbins v2.0.0 (Croucher et al., 2015) was used to identify recombinant regions of the genome which were subsequently masked for phylogenetic analysis. Pseudosequences of polymorphic positions were used to create maximum likelihood trees using RAxML v8.1.17 (Stamatakis, 2014). De novo assembly was carried out using Spades 3.5.0 using '-careful' and ' -k 21,33,55,65,77,83,91' options (Bankevich et al., 2012).

To proactively detect outbreaks from WGS data, SNP typing was carried out on $S$. flexneri isolates belonging to clonal complex (CC) 245 and CC145. CC145 mostly comprises $S$. boydii serotypes but includes $S$. flexneri serotype 6 , as this $S$. flexneri serotype was misidentified historically (Wirth et al., 2006; Chattaway et al., 2017). Hierarchical single linkage clustering was performed at seven descending thresholds of SNP distance $(\Delta 250, \Delta 100, \Delta 50, \Delta 25, \Delta 10, \Delta 5, \Delta 0)$ as previously described (Dallman et al., 2016). This clustering results in a discrete seven digit code where each number represents the 
cluster membership at each descending SNP distance threshold. The resultant SNP addresses describes an isolates position in the $S$. flexneri population structure where two isolates with the same SNP addresses have 0 SNP differences between them.

\section{RESULTS}

\section{Demographic of Patients}

There were 330 isolates reported between 1st August 2015 and 18th January 2016, 236 (71\%) were from males, 85 (26\%) from females, and for nine $(3 \%)$ cases the sex was not stated. Travel history was not provided for 154 (47\%) cases, 85 cases reported travel 7 days prior to onset of symptoms and 91 (27\%) reported that they did not travel during the 7 days prior to onset of symptoms. The most frequently reported destinations were India $(n=16,5 \%)$ and Pakistan $(n=11,3 \%)$ with other counties accounting for less $<1 \%$ each.

\section{Comparison of WGS Predicted Serotype versus PCR Predicted Serotype Results}

Of the 330 cultures tested prospectively by WGS, 306 (93\%) had concordant results with phenotypic serotyping. Three of the mismatched results between the phenotypic and genotypic tests were attributed to mutations identified in $\mathrm{O}$-antigen synthesis or modification genes (Table 1), including nonsense mutations resulting in early stop codons $(n=2)$ and a frameshift mutation. Repeat testing of sample 4 revealed the discrepancy was due to an auto-agglutination reaction of the strain with the sera (Table 1).

The remaining mismatches were novel serotype gene profiles detected by WGS, both associated with outbreaks (Cluster 2, $n=13$; Cluster 4, $n=5$ ) (Tables 1, 2). Cluster 2 comprised 13 isolates associated with a local community outbreak. The isolates failed to agglutinate any of the serotype specific S. flexneri antisera and were positive for wzx1-5 and gtrIc gene targets in the PCR but were negative for
gtrI. Typical strains of serotype 1c are positive for $w z x 1$, gtrI, and gtrIc (Supplementary Table S1). These strains were designated $1 \mathrm{c}$ variants $(1 \mathrm{cv})$. Cluster 4 comprised five isolates of $S$. flexneri associated with an outbreak of gastrointestinal symptoms in five captive chimpanzees phenotypically identified as serotype $3 \mathrm{a}$. The isolates had $w z x 1-5$, oac, and grtX but were also positive for gtrII and were designated $3 a$ variant (3av).

\section{Outbreak Investigation Using SNP Clustering Typing}

Analysis of the WGS data organized 161 of the 330 isolates into 36 five SNP single-linkage clusters of two or more isolates associated with CC245 and one cluster in CC145. The median number of cases in these clusters was 2 and ranged from 2 to 27; 32 (89\%) clusters investigated comprised less than 5 isolates (Table 2). It was not possible to identify epidemiological links associated with these small clusters using only the limited epidemiological data available from laboratory report forms. However, 9/32 (28\%) comprised at least one case reporting recent travel abroad prior to onset of symptoms.

During the period of the study, four outbreaks were identified following routine surveillance of local hospital reports of gastrointestinal symptoms caused by $S$. flexneri. SNP typing confirmed that all the isolates belonging to each outbreak cluster were closely related and monophyletic. Cluster 1 was the largest cluster and had a high male to female ratio with the $97 \%$ of cases being adult males (for two cases the gender was not stated) (Table 2). Isolates from this cluster were observed throughout the study period. The minimum SNP distance between isolates in this cluster was zero with the median distance 17. The maximum SNP distance between any two isolates was 64, however the majority of these SNPs were caused by a transposase mediated recombination of pic, encoding a serine protease. This demographic of adult males has previously been shown to be characteristic of clusters linked to sexual transmission among the MSM community (Borg et al., 2012; Gilbart et al., 2015; Baker et al., 2015; Simms et al., 2015). Cluster 1 was part of larger outbreak of

TABLE 1 | Summary of mismatched phenotypic and genotypic results.

\begin{tabular}{|c|c|c|c|c|c|}
\hline Reference no. & Sample no. & Phenotypic serotype & PCR serotype & WGS serotype & Explanation of mismatch \\
\hline SRR4787737 & 1 & $1 a$ & $1 b$ & $1 b$ & $\begin{array}{l}\text { Oaclb contains an early stop codon at } \\
\text { position } 128 \text {, resulting in the } 1 \text { a phenotype }\end{array}$ \\
\hline SRR4786841 & 2 & $X$ variant $/ Y$ variant & $3 a$ & $3 a$ & $\begin{array}{l}\text { Oac contains an early stop codon at } \\
\text { position } 20 \text { resulting in the } X \text { phenotype }\end{array}$ \\
\hline SRR5018321 & 3 & $Y$ variant & $2 a$ & $2 a$ & $\begin{array}{l}\text { Frameshift mutation (insertion of two A's) at } \\
\text { position } 1116 \text { rendering gtrll non-functional } \\
\text { and resulting in the } Y \text { phenotype }\end{array}$ \\
\hline SRR5017471 & 4 & $X$ variant & Y & Y & $\begin{array}{l}\text { Auto agglutination strain resulting in } \\
\text { incorrect phenotype }\end{array}$ \\
\hline SRR4788187 SRR4897598 & $5-6$ & $1 c$ & $1 c$ & $1 a$ & $\begin{array}{l}\text { No explanation for the mismatch could be } \\
\text { determined }\end{array}$ \\
\hline Cluster 2 (13 isolates) & $7-19$ & Negative serology & $1 \mathrm{cv}$ & $1 \mathrm{cv}$ & Novel serotype \\
\hline Cluster 4 (five isolates) & $20-24$ & $3 a$ & $3 a v$ & $3 a v$ & Novel serotype \\
\hline
\end{tabular}

Clusters 2 and 4 contain multiple isolates, please see Supplementary Table S2 for the Reference numbers. 
TABLE 2 | Summary of Clusters detected by WGS.

\begin{tabular}{|c|c|c|c|c|c|c|c|c|c|c|}
\hline $\begin{array}{l}\text { Cluster } \\
\text { no. }\end{array}$ & $\begin{array}{c}\text { No. } \\
\text { cases }\end{array}$ & Travel & $\begin{array}{l}\text { M:F } \\
\text { ratio }\end{array}$ & $\begin{array}{c}\text { Age } \\
\text { range }\end{array}$ & Serotype & $\begin{array}{l}\text { Epidemiological } \\
\text { context/transmission } \\
\text { route }\end{array}$ & SNP address & $\begin{array}{l}\text { Minimum } \\
\text { SNP } \\
\text { difference }\end{array}$ & $\begin{array}{l}\text { Maximum } \\
\text { SNP } \\
\text { difference }\end{array}$ & $\begin{array}{l}\text { Median } \\
\text { SNP } \\
\text { difference }\end{array}$ \\
\hline 1 & 121 & 7 & 119:0* & $20-63$ & $2 a$ & MSM & 34.42.42.42.\# & 0 & $40^{\wedge}$ & 17 \\
\hline 2 & 13 & 0 & $4: 9$ & $23-96$ & $\$ 1 \mathrm{cV}$ & Community & 3.45 .45 .46 .46 .46 .47 & 0 & 0 & 0 \\
\hline 3 & 7 & 1 & $3: 4$ & $21-53$ & $2 b$ & Restaurant & 8.9.9.138.140.154.167 & 0 & 0 & 0 \\
\hline 4 & 5 & N/A & N/A & N/A & \$3av & Captive chimpanzees & 45.130.197.328.353.405.\# & 1 & 4 & 2.5 \\
\hline
\end{tabular}

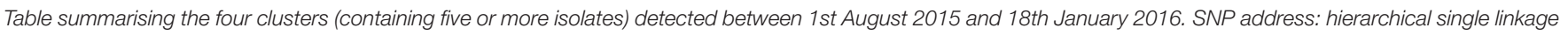

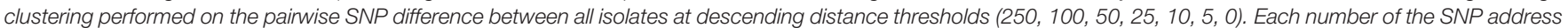

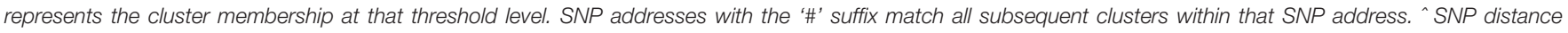

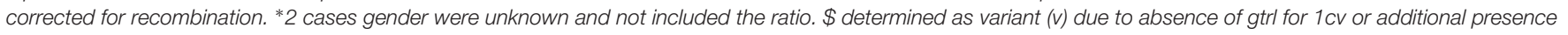
of gtrll for 3av.

S. flexneri serotype 2 a previously described by Simms et al. (2015).

Clusters 2 and 3 were community outbreaks and cases were geographically linked. They were temporally restricted and genetically homogenous, exhibiting 0-1 SNPs difference in the core genome between isolates. Despite a thorough epidemiological investigation, the source and route of transmission associated with Cluster 2 could not be determined. Cluster 3 was linked to consumption of contaminated food at a restaurant, most likely due to an infected food handler. Cluster 4 was associated with an outbreak of gastrointestinal symptoms in a group of captive chimpanzees with a minimum SNP distance between isolates of one and a maximum SNP distance of four.

The S. flexneri population structure clusters into seven distinct phylogenetic groups (PGs) (Connor et al., 2015). Phylogenetic analysis of the diversity of CC245 in the PHE collection is shown in Figure 1. Four out of seven clades are represented by samples received by PHE through routine surveillance. With respect to the clusters described in this study, Clusters 1, 3, and 4 fall within PG3 and Cluster 2 falls within PG1.

\section{DISCUSSION}

This study showed WGS to be a robust and reliable method for serotyping $S$. flexneri isolates and provided additional strain discrimination at the SNP level. There was high correlation between phenotypic serotyping and WGS serotyping (93\%) thus facilitating the prospective comparison of WGS data with historical phenotypic data and ensuring continuity for monitoring trends in the incidence of different serotypes over time.

In three of the mismatches, the WGS derived serotype was shown to predict serotype based on gene presence which may not be expressed phenotypically. WGS provided insight on the effect of mutations on $\mathrm{O}$-antigen modification genes and potential mechanisms that inactivate phenotypic expression. WGS analysis also identified novel serotype gene profiles associated with two outbreaks.

Serotype is not a robust phylogenetic marker, as the $\mathrm{O}$-antigen synthesis/modification genes are encoded on mobile genetic

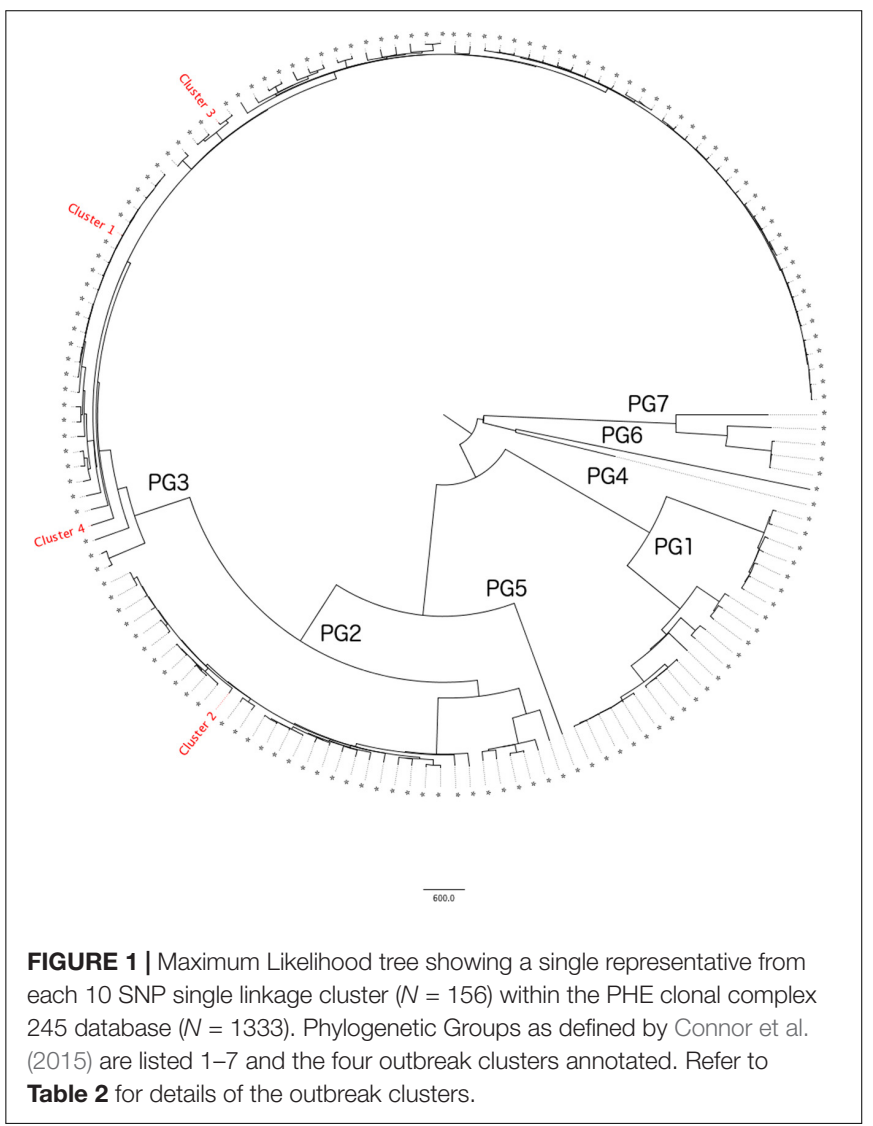

elements (prophages) (Connor et al., 2015). Prior to the implementation of WGS, detection of outbreaks of S. flexneri at $\mathrm{PHE}$ relied on the identification of epidemiological links between cases as serotyping was not discriminatory enough to detect outbreaks during routine surveillance. During this study, SNP typing provided microbiological evidence that the isolates associated with each of the four outbreaks identified were closely related. SNP typing has been used previously to investigate outbreaks of $S$. sonnei, and this study provides further evidence of the utility of this approach $(\mathrm{McD}$ onnell et al., 2013; Dallman et al., 2016; Mook et al., 2016; Baker et al., 2017). 
Three of the outbreak clusters were temporally and genetically restricted with less than five SNP differences observed between outbreak isolates. In contrast, an outbreak representing on-going person-to-person transmission within the MSM community exhibited between zero and 64 SNP differences between isolates. S. flexneri may become endemic in defined populations or communities, such as religious communities or sexual networks. Over time circulating strains will accumulate mutations leading to an increase in observed SNPs between cases in that network. Similarly, extended transmission provides greater opportunities for horizontal gene transfer in the population and therefore bioinformatic analyses have to be robust to such influxes of variation.

This heterogeneity in genetic conservation between epidemiologically linked cases highlights the need to be flexible with respect to the case cluster definition. Epidemiological data associated with each outbreak, and WGS analyses of the deeper phylogenetic relationship between isolates, should be used in concert to inform outbreak investigations.

The use of WGS for routine surveillance of S. flexneri provides reliable and robust data that can be used to monitor trends in the incidence of different serotypes over time. WGS derived serotyping data ensures backward compatibility with historical phenotypic serotyping data. SNP typing can be used to facilitate outbreak investigations in real-time thereby enhancing surveillance strategies and providing the opportunities for implementing rapid public health interventions.

\section{INFORMED CONSENT}

Informed consent was not required as all data in this study was anonymized.

\section{REFERENCES}

Baker, K. S., Campos, J., Pichel, M., Della Gaspera, A., Duarte-Martínez, F., Campos-Chacón, E., et al. (2017). Whole genome sequencing of Shigella sonnei through PulseNet Latin America and Caribbean: advancing global surveillance of foodborne illnesses. Clin. Microbiol. Infect. doi: 10.1016/j.cmi.2017. 03.021 [Epub ahead of print].

Baker, K. S., Dallman, T. J., Ashton, P. M., Day, M., Hughes, G., Crook, P. D., et al. (2015). Intercontinental dissemination of azithromycin-resistant shigellosis through sexual transmission: a cross-sectional study. Lancet Infect. Dis. 15, 913-921. doi: 10.1016/S1473-3099(15)00002-X

Bankevich, A., Nurk, S., Antipov, D., Gurevich, A. A., Dvorkin, M., Kulikov, A. S., et al. (2012). SPAdes: a new genome assembly algorithm and its applications to single-cell sequencing. J. Comput. Biol. 19, 455-477. doi: 10.1089/cmb.2012. 0021

Bolger, A. M., Lohse, M., and Usadel, B. (2014). Trimmomatic: a flexible trimmer for Illumina sequence data. Bioinformatics 30, 2114-2120. doi: 10.1093/bioinformatics/btu170

Borg, M. L., Modi, A., Tostmann, A., Gobin, M., Cartwright, J., Quigley, C., et al. (2012). Ongoing outbreak of Shigella flexneri serotype 3 a in men who have sex with men in England and Wales, data from 2009-2011. Euro Surveill. 17:20137.

Chattaway, M. A., Schaefer, U., Tewolde, R., Dallman, T. J., and Jenkins, C. (2017). Identification of Escherichia coli and Shigella species from whole-genome sequences. J. Clin. Microbiol. 55, 616-623. doi: 10.1128/JCM.01790-16

Connor, T. R., Barker, C. R., Baker, K. S., Weill, F. X., Talukder, K. A., Smith, A. M., et al. (2015). Species-wide whole genome sequencing reveals historical

\section{AUTHOR CONTRIBUTIONS}

DG and AG performed the DNA extractions, PCR, phenotypic serotyping and identification. $\mathrm{MC}$ and CJ implemented the wet lab WGS pipelines and performed analysis. TD, $\mathrm{HH}$, and DG performed bioinformatic analysis. MC and CJ wrote the manuscript and TD, DG, and $\mathrm{HH}$ contributed to the manuscript.

\section{FUNDING}

This work was supported by the National Institute for Health Research Health Protection Research Unit in Gastrointestinal Infections (\#109524). The views expressed are those of the author(s) and not necessarily those of the NHS, the NIHR, the Department of Health or Public Health England.

\section{ACKNOWLEDGMENT}

We would like to thank Vivienne do Nascimento and Dawn Hedges for curation of the serotyping scheme in GBRU.

\section{SUPPLEMENTARY MATERIAL}

The Supplementary Material for this article can be found online at: http://journal.frontiersin.org/article/10.3389/fmicb. 2017.01700/full\#supplementary-material

TABLE S1 | Serotype by gene presence.

TABLE S2 | Sample list and results.

global spread and recent local persistence in Shigella flexneri. eLife 4:e07335. doi: 10.7554/eLife.07335

Croucher, N. J., Page, A. J., Connor, T. R., Delaney, A. J., Keane, J. A., Bentley, S. D., et al. (2015). Rapid phylogenetic analysis of large samples of recombinant bacterial whole genome sequences using Gubbins. Nucleic Acids Res. 43, e15. doi: 10.1093/nar/gku1196

Dallman, T. J., Byrne, L., Ashton, P. M., Cowley, L. A., Perry, N. T., Adak, G., et al. (2015). Whole-genome sequencing for national surveillance of Shiga toxinproducing Escherichia coli O157. Clin. Infect. Dis. 61, 305-312. doi: 10.1093/ cid/civ318

Dallman, T. J., Chattaway, M. A., Mook, P., Godbole, G., Crook, P. D., and Jenkins, C. (2016). Use of whole-genome sequencing for the public health surveillance of Shigella sonnei in England and Wales, 2015. J. Med. Microbiol. 65, 882-884. doi: 10.1099/jmm.0. 000296

Gentle, A., Ashton, P. M., Dallman, T. J., and Jenkins, C. (2016). Evaluation of molecular methods for serotyping Shigella flexneri. J. Clin. Microbiol. 54, 1456-1461. doi: 10.1128/JCM.03386-15

Gilbart, V. L., Simms, I., Jenkins, C., Furegato, M., Gobin, M., Oliver, I., et al. (2015). Sex, drugs and smart phone applications: findings from semistructured interviews with men who have sex with men diagnosed with Shigella flexneri 3a in England and Wales. Sex Transm. Infect. 91, 598-602. doi: 10.1136/sextrans2015-052014

Gross, R. J., and Rowe, B. (1985). "Serotyping of Escherichia coli”, in The Virulence of Escherichia coli, ed. M. Sussman (Cambridge: Cambridge University Press), $345-360$. 
Kotloff, K. L., Winickoff, J. P., Ivanoff, B., Clemens, J. D., Swerdlow, D. L., Sansonetti, P. J., et al. (1999). Global burden of Shigella infections: implications for vaccine development and implementation of control strategies. Bull. World Health Organ. 77, 651-666.

Langmead, B., and Salzberg, S. L. (2012). Fast gapped-read alignment with Bowtie 2. Nat. Methods 9, 357-359. doi: 10.1038/nmeth.1923

Li, H., and Durbin, R. (2010). Fast and accurate long-read alignment with BurrowsWheeler transform. Bioinformatics 26, 589-595. doi: 10.1093/bioinformatics/ btp698

Li, H., Handsaker, B., Wysoker, A., Fennell, T., Ruan, J., Homer, N., et al. (2009). The sequence alignment/map format and SAMtools. Bioinformatics 25, 2078-2079. doi: 10.1093/bioinformatics/btp352

McDonnell, J., Dallman, T., Atkin, S., Turbitt, D. A., Connor, T. R., Grant, K. A., et al. (2013). Retrospective analysis of whole genome sequencing compared to prospective typing data in further informing the epidemiological investigation of an outbreak of Shigella sonnei in the UK. Epidemiol. Infect. 141, 2568-2575. doi: 10.1017/S0950268813000137

McKenna, A., Hanna, M., Banks, E., Sivachenko, A., Cibulskis, K., Kernytsky, A., et al. (2010). The genome analysis toolkit: a MapReduce framework for analyzing next-generation DNA sequencing data. Genome Res. 20, 1297-1303. doi: $10.1101 /$ gr.107524.110

Mook, P., McCormick, J., Bains, M., Cowley, L. A., Chattaway, M. A., Jenkins, C., et al. (2016). ESBL-producing and macrolide-resistant Shigella sonnei infections among men who have sex with men, England, 2015. Emerg. Infect. Dis. 22, 1948-1952. doi: 10.3201/eid2211.160653

Simms, I., Field, N., Jenkins, C., Childs, T., Gilbart, V. L., Dallman, T. J., et al. (2015). Intensified shigellosis epidemic associated with sexual transmission in men who have sex with men-Shigella flexneri and S. sonnei in England, 2004 to end of February 2015. Euro Surveill. 20, 21097.

Stamatakis, A. (2014). RAxML version 8: a tool for phylogenetic analysis and post-analysis of large phylogenies. Bioinformatics 30, 1312-1313. doi: 10.1093/ bioinformatics/btu033

Sun, Q., Knirel, Y. A., Lan, R., Wang, J., Senchenkova, S. N., Jin, D., et al. (2012a). A novel plasmid-encoded serotype conversion mechanism through addition of phosphoethanolamine to the O-antigen of Shigella flexneri. PLOS ONE 7:e46095. doi: 10.1371/journal.pone.0046095
Sun, Q., Lan, R., Wang, Y., Wang, J., Xia, S., Wang, Y., et al. (2012b). Identification of a divergent $O$-acetyltransferase gene $o a c_{1 b}$ from Shigella flexneri serotype 1b strains. Emerg. Microbes Infect. 1, e21. doi: 10.1038/emi. 2012.22

Sun, Q., Lan, R., Wang, Y., Zhao, A., Zhang, S., Wang, J., et al. (2011). Development of a multiplex PCR assay targeting $\mathrm{O}$-antigen modification genes for molecular serotyping of Shigella flexneri. J. Clin. Microbiol. 49, 3766-3770. doi: 10.1128/ JCM.01259- 11

von Seidlein, L., Kim, D. R., Ali, M., Lee, H., Wang, X., Thiem, V. D., et al. (2006). A multicentre study of Shigella diarrhoea in six Asian countries: disease burden, clinical manifestations, and microbiology. PLOS Med. 3:e353. doi: 10.1371/journal.pmed.0030353

Wei, J., Goldberg, M. B., Burland, V., Venkatesan, M. M., Deng, W., Fournier, G., et al. (2003). Complete genome sequence and comparative genomics of Shigella flexneri serotype 2a strain 2457T. Infect. Immun. 71, 2775-2786. doi: 10.1128/ IAI.71.5.2775-2786.2003

Wirth, T., Falush, D., Lan, R., Colles, F., Mensa, P., Wieler, L. H., et al. (2006). Sex and virulence in Escherichia coli: an evolutionary perspective. Mol. Microbiol. 60, 1136-1151. doi: 10.1111/j.1365-2958.2006. 05172.x

Zhang, S. X., Wang, J. P., Jin, D., Chen, D. L., Liu, K., Wang, Y. T., et al. (2012). Development of a singleplex PCR assay targeting $\mathrm{O}$-antigen modification genes for molecular serotyping of Shigella flexneri. Zhonghua Liu Xing Bing Xue Za Zhi 33, 328-331.

Conflict of Interest Statement: The authors declare that the research was conducted in the absence of any commercial or financial relationships that could be construed as a potential conflict of interest.

Copyright (c) 2017 Chattaway, Greig, Gentle, Hartman, Dallman and Jenkins. This is an open-access article distributed under the terms of the Creative Commons Attribution License (CC BY). The use, distribution or reproduction in other forums is permitted, provided the original author(s) or licensor are credited and that the original publication in this journal is cited, in accordance with accepted academic practice. No use, distribution or reproduction is permitted which does not comply with these terms. 